L'Assemblée des délégués de la FMH a exprimé son soutien à la stratégie MNT dans une prise de position qui précise que le succès du deuxième paquet de mesures "Prévention dans le domaine des soins" est indissociable de certaines conditions-cadres. Ces dernières se détérioreront massivement si les limitations de temps des positions tarifaires "Consultation", "Visite» et "Travaux en l'absence du patient" prévues dès 2018 par la deuxième intervention du Conseil fédéral sont maintenues. De telles limitations pénaliseront systématiquement les groupes de patients vulnérables. Du point de vue de la médecine et de la santé publique, il existe pourtant des possibilités d'améliorer l'efficacité, l'adéquation et l'économicité sans mettre en danger les patients, la qualité des soins et la santé publique. Pour y parvenir, il faut que les conditions-cadres figurant dans la prise de position soient prises en compte car elles ont été élaborées par des médecins et des spécialistes en santé publique qui, jour après jour, s'engagent avec les patients pour le bien de ces derniers.

\title{
Stratégie nationale Prévention des maladies non transmissibles
}

\author{
Assemblée des délégués de la FMH
}

Dans un avenir proche, la prévention des maladies non transmissibles (MNT) aura atteint une nouvelle dimension dans le système de santé grâce à la stratégie nationale et à son plan de mesures. Sa diffusion parmi les médecins et l'engagement de ces derniers permettra d'étendre son effet à la population. Une collaboration optimale de tous les acteurs est incontournable. Cette association implique une sensibilisation du corps médical et des milieux politiques.

\section{Contexte}

Début 2016, le dialogue «Politique nationale de la santé», le forum permanent de la Confédération et des cantons, et le Conseil fédéral ont adopté la stratégie MNT. Diverses impulsions de la part du corps médical, mais surtout les coûts croissants des primes et l'augmentation attendue des maladies non transmissibles due notamment à l'évolution sociale et démographique de la population suisse ont eu un effet déclencheur. Le plan de mesures MNT prévoit les trois grandes lignes suivantes:

- Promotion de la santé et prévention destinées à la population - développer les programmes cantonaux de prévention et de promotion de la santé

- Prévention dans le domaine des soins - améliorer les interfaces entre la prévention et les soins

- Prévention dans l'économie et le monde du travail renforcer les conditions-cadres propices à la santé en collaboration avec l'économie

La stratégie MNT et son plan de mesures soulignent le rôle majeur des prestations du médecin dans le do- maine de la promotion de la santé et de la prévention. Ceci implique un positionnement clair de la part du corps médical puisque c'est notamment la diffusion de cette thématique parmi les médecins qui permettra d'étendre son effet à la population. Ces derniers ont en effet accès à la population de tous âges et de tous milieux sociaux dans les quatre coins du pays et bénéficient de sa confiance, élément central de motivation et d'adhésion. Le corps médical fournit ainsi des prestations pertinentes dans les trois grandes lignes susmentionnées et dans toutes les phases de la vie. Pour le corps médical, la deuxième mesure «Prévention dans le domaine des soins» est particulièrement importante. Le terrain pour encourager la prévention par les médecins est préparé depuis longtemps. Les projets «Coaching Santé» du CMPR, Vivre sans tabac, Paprica et EviPrev sont des exemples de projets d'excellence en matière de santé publique. La prévention est inhérente aux soins de santé primaires en particulier. Les spécialistes agissent eux aussi dans le domaine de la prévention, avant tout au niveau des groupes de patients et de maladies couvertes par leur spécialité, mais aussi 
au niveau de la population. Un rôle particulier revient aux médecins qui exercent dans les domaines psychiatrique, pédiatrique ou gynécologique car ils sont souvent le seul référent médical de leurs patients.

\section{Arguments}

\section{Accès à la population dans toutes les phases de la vie}

Les occasions de parler de comportement préventif avec les patients et de les motiver à changer sont les plus nombreuses chez les médecins dans la mesure où près de $80 \%$ de la population adulte consulte au moins une fois par an un médecin installé. Les contacts médicaux sont particulièrement efficaces chez les enfants: les consultations préventives en pédiatrie sont primordiales pour transmettre des informations importantes, qu'il s'agisse de promotion de la santé ou, à long terme, d'influencer le plus favorablement possible le comportement des jeunes vis-à-vis de leur santé. La promotion de la santé et la prévention sont des processus de toute une vie qui commencent avant la naissance.

\section{Interface prévention/soins dans le cadre de l'activité médicale}

En fonction des attentes du patient et dans le sens d'une prise en charge holistique, le médecin (de famille) peut aborder des aspects de promotion de la santé et de prévention primaire, secondaire, tertiaire ou quaternaire lors d'une consultation, et ce parallèlement aux questions sur le diagnostic, le traitement, la réadaptation ou les soins palliatifs. Il ne s'agit pas uniquement d'émettre des recommandations et que celles-ci soient comprises, mais elles doivent être applicables, soutenues et encouragées par la société.

\section{Collaboration interdisciplinaire}

Bien entendu, le corps médical ne pourra pas à lui seul mettre en œuvre toutes les mesures. Les attentes globales et le point de vue systémique exigent de plus en plus une conception interdisciplinaire du travail et l'application de méthodes issues de différentes disciplines. Dans le cabinet médical, les assistantes médicales (CFC) et les coordinatrices en médecine ambulatoire (CMA) sont particulièrement prédestinées, au terme d'une formation adéquate, aux activités de conseil et il s'agit de les encourager dans cette voie. Les autres groupes professionnels du domaine de la santé et les ligues de santé doivent absolument intégrer ces réseaux. Pour répondre à une utilisation optimale des ressources, il importe que les médecins puissent mettre en place une gestion des interfaces de bonne qualité, ce qui demande de prévoir du temps et de l'argent.

\section{Approche participative}

Les patients doivent être au centre du traitement. De ce fait, ils participent aux décisions sur leur traitement en tant que partenaires paritaires. Le médecin est l'expert pour toutes les questions médicales tandis que le patient connaît ses propres valeurs, ses souhaits et ses convictions. C'est au médecin d'établir une relation de confiance et d'inciter les échanges sur le thème de la prévention en posant les questions adéquates. Cette approche participative (une exigence depuis Ottawa 1986) crée aussi l'acceptation pour l'application de mesures. Certains groupes de population sont plus particulièrement menacés par les MNT: le niveau de formation peu élevé et la migration engendrent par exemple davantage de surpoids, de déséquilibre alimentaire ou une consommation de tabac élevée, et un recours aux consultations de prévention plutôt plus rare.

\section{Conditions-cadres financières et politiques}

Les mesures planifiées et les améliorations qu'elles visent ne seront pas gratuites et elles ne peuvent pas non plus être dissociées d'autres questions de politique de santé ni des attentes de la société. Le plan de mesures repose sur un système fédéraliste caractérisé par une multitude d'acteurs et de mesures, dont le financement est fragmenté.

\section{Nos revendications}

- Les consultations médicales sont à considérer comme un instrument central pour toutes les formes de prévention afin de créer à tout âge des conditions propices à la santé au niveau du patient et de la population.

- Les médecins demandent une approche holistique de tous les thèmes pertinents en matière de prévention (dépendance, santé psychique, MNT, etc.). La prévention et la promotion de la santé font l'objet d'une démarche globale au quotidien et non de piliers thématiques.

- La recherche sur les soins doit fournir des données scientifiques pour toutes les formes de prévention en tenant compte des interfaces.

- La rémunération des prestations médicales du domaine de la promotion de la santé et de la prévention est à garantir et à réglementer de manière tarifaire en vue d'une application durable dans le cadre d'une approche économique coûts globauxutilité. 
- Pour s'imposer au cabinet médical, les approches consultatives doivent non seulement être développées par des médecins, mais également mises en œuvre et diffusées par les sociétés de discipline médicale et leurs structures.

- Le corps médical est appelé à utiliser les instruments d'excellence existants. II est nécessaire de les faire connaître et de les rendre facilement accessibles dans le cadre de la stratégie MNT.

- L'approche participative doit placer les patients au centre du traitement. Au vu des besoins spécifiques de certains groupes de population, les possibilités d'adapter les mesures ne doivent pas être oubliées afin de permettre aux médecins d'exercer la prévention aussi parmi ces groupes (p. ex. financement d'interprètes interculturels).

FMH

division Santé publique

Elfenstrasse 18

CH-3000 Berne 15

public.health[at]fmh.ch
- Les médecins mènent des activités de prévention et de promotion de la santé selon une approche similaire à un réseau de soins interprofessionnels et intégrés et représentent, avec les autres professionnels de santé, un partenaire importan dans la mise en œuvre de la stratégie MNT.

- Le corps médical soutient le développement et I'intégration des approches d'autres professions de la santé. Ces approches doivent toutefois être développées, mises en place et diffusées en collaboration avec leurs organisations professionnelles et dans le cadre de leurs structures.

- II ne s'agit pas uniquement d'émettre des recommandations et que celles-ci soient comprises, mais elles doivent être applicables, soutenues et encouragées par la société. La prévention structurelle est une composante obligatoire de la mise en œuvre.

Organe suprême de consultation: Assemblée des délégués de la FMH, Berne, 29 juin 2017 